\title{
VIVERE PER MORIRE O MORIRE PER VIVERE L'ARCHETIPO DELLA MORTE PRECOCE NELLE TRADIZIONI EROICHE
}

\author{
Alvaro Barbieri \\ Università degli Studi di Padova
}

RIASSUNTO: Ponendo in triangolazione le pratiche militari del mondo premoderno, le ideologie delle "società di vergogna" (shame societies) e i plessi simbolici della poesia eroica universale, il presente articolo riattraversa il tema cruciale della morte precoce - subitanea rossa violenta -, momento di densità rivelativa mediante il quale il guerriero d'élite compie sé stesso e modella il finale della propria vita. Morire anzitempo sul campo di battaglia è il modo con cui il combattente sconfigge la mortalità ordinaria, sottraendosi una volta per tutte all'avvizzimento della carne. Vista in tale prospettiva, la morte precoce si pone come una delle prestazioni tramite le quali l'eroe milita contro le forze del preformale e del caos indifferenziato.

PAROLE CHIAVE: caducità, eroismo, guerra, morte, onore, autenticità

ABSTRACT: Triangulating the military practices of the pre-modern world, the ideologies of "shame societies" and the symbolic plexuses of universal heroic poetry, this article revisits the crucial theme of early death - sudden violent red -, a moment of revelatory density through which the elite warrior accomplishes himself and shapes the end of his life. Dying before his time on the battlefield is the fighter's way of defeating ordinary mortality by escaping the withering of the flesh once and for all. Seen from this perspective, premature death is one of the performances through which the hero militates against the forces of the pre-formal and undifferentiated chaos.

KEY-WORDS: Transience, Heroism, War, Death, Honour, Authenticity 
[...] in ultima analisi, l'eroismo è un principio del corpo e partecipa infine al contrasto tra la robustezza del corpo e la distruzione della morte (Mishima Yukio, Sole e acciaio)

[C]ome hanno ripetuto spesso i comandanti talebani negli ultimi anni, «non si può essere sconfitti da chi non è disposto a morire» (Gastone Breccia, Missione fallita. La sconfitta dell'Occidente in Afghanistan)

Lì [in America Latina] il valore della vita umana è molto diverso.

Flirtano con la morte in un modo che non conosciamo.

(Eugenio Viola, L'inviato speciale [intervista apparsa su «D Lui», dicembre 2021])

Se c'è una congiuntura in cui l'uomo entra drammaticamente in tensione con la coscienza della propria finitudine, cercando parimenti le occasioni e gli strumenti rituali per trascendersi ed eternarsi, quella è senz'altro la condizione di guerra o, per essere più precisi, il combattimento.

La battaglia, assieme alla sineddoche duellistica che ne singolarizza pars pro toto le caratteristiche, ${ }^{1}$ è il luogo dell'estremo e dell'assoluto, un'esperienza del sacro sinistro (o di trasgressione $)^{2}$ che potenzia a dismisura il senso dell'esistere e dell'esserci proprio là dove si sente battere più forte l'ala della morte. Quando il rischio è massimo, la vita appare come aumentata e intensificata, attraversata da un battito potente che fa percepire in un regime esasperato dei sensi il mistero e il soprassalto affettivo dell'esserci. È il grande paradosso della densità esperienziale: dove la corporeità è più gracile ed esposta al "terrore della storia", ${ }^{3}$ dove si avverte nel modo più disarmante la fugacità del transito terrestre e

${ }^{1}$ Nelle culture di guerra tradizionali, duello e battaglia sono modalità di combattimento apparentate da vari
tratti comuni: la contesa si disputa aperto Marte, in contesto diurno, spesso in un luogo e in un tempo con-
venuti tra le due parti, con una forte aura di ritualità e un'enfasi speciale sul valore destinale e ordalico dell'af-
frontamento, di cui si sottolinea la natura dirimente e decisiva. Alla luce di queste omologie, credo non sia
azzardato affermare che in molti casi la singolar tenzone si presenta come una sorta di battaglia in miniatura,
mentre la battaglia può apparire alla stregua di un duello espanso (collettivo), dilatato su larga scala.
${ }^{2}$ Per l'enucleazione di questa categoria antropologico-religiosa, cfr. CAILLOIS 2001: 89-118.
${ }^{3}$ Prelevo l'espressione e la categoria dall'opera di Mircea Eliade (si veda in particolare ELIADE 1968: 177-186). 
Vivere per morire o morire per vivere: l'archetipo della morte precoce nelle tradizioni eroiche

la natura momentanea del nostro stare al mondo, proprio là si costella l'archetipo dell'autenticità e la vita ci pare più vita che altrove. Appesi al nulla e sempre sul punto di precipitarvi in una repentina dissolvenza a nero, i soldati immersi nel turbine della battaglia sperimentano la tensione estrema della vita che si libra incerta sull'abisso. ${ }^{4}$ Nella morale eroica, l'uomo è compiutamente sé stesso solo quando si trova a un passo dalla perdita di sé, sulle soglie della fine, in bilico tra essere e non esser più. In questa prospettiva, l'action d'éclat appare tanto più ricca di significato quanto più grande è il rischio che comporta: costeggiando dappresso la propria dissoluzione, il campione militare riempie di significato la sua impresa. Per i guerrieri delle società arcaiche, la tensione euforizzante dello scontro è il modo con cui ci si accosta alla bellezza e all'intensità dell'essere. Osservato nella totalità delle sue implicazioni, il combattimento non ci appare più come una semplice manifestazione di forza guerriera, un mero sfiato di violenza o una pratica professionale, ma come un modo plenario di percepire la propria vita nel confronto (e nello scontro) con l'esistente, ovvero come un sentimento incondizionato del proprio essere nel mondo. Nel parossismo della lotta gli uomini sperimentano la paura dell'annullamento, la coscienza acutissima della propria transitorietà, il giubilo dell'esistenza in sé, lo scandalo e la potenza della vita, il miracolo degli stati di grazia e delle sensazioni più accese. Perciò la battaglia, al picco dell'acuzie e al colmo del rischio di morte, può essere pensata e sperimentata come il luogo di verità per eccellenza: una manifestazione piena $\mathrm{e}$ totale, una sovrabbondanza di vita scaturita dalla contiguità col suo contrario. Le culture dell'onore attribuiscono un carisma speciale e un appeal sociale di ordine superiore agli uomini che rischiano senza esitare il tutto per tutto, agli asceti delle armi che si mettono

\footnotetext{
${ }^{4}$ Dopo gli squilli sopracuti del combattimento, ogni altra manifestazione della vita si abbassa di volume. Appetto ai furori della lotta, il tempo di pace appare scialbo e depressivo, routinario e ripiegato sulle cadenze spente della quotidianità e dell'esistenza comune. Da questo divario di intensità esperienziale (oltre che dalle conseguenze dei traumi prodotti dagli scontri e dagli orrori della guerra) discende il tipico disagio del reduce, ben noto in letteratura psicologica e tematizzato negli intrecci di numerosi film recenti. Drogato di guerra, incapace di adattarsi al grigiore e alla prosa del mondo ordinario, il veterano trova momenti di condivisione e di sintonia solo coi vecchi lupi, i fratelli d'armi con cui ha condiviso i momenti estremi del fuoco e del sangue, mentre i valori, gli stili di vita e il modo d'essere dei civili gli appaiono estranei, privi di valore e persino incomprensibili. Tra le pellicole che descrivono e problematizzano con maggior senso critico e con più capacità di rappresentazione la sindrome di isolamento e l'alienazione dei reduci, menzionerei almeno The Hurt Locker di Kathryn Ann Bigelow (Usa 2008) e Billy Lynn - Un giorno da eroe (Lynn's Long Halftime Walk) di Ang Lee (Usa/GB 2016).
} 
in gioco ogni volta sul filo delle lame, esponendo la propria carne all'alea e alla fatalità dello scontro. Il canone eroico predilige chi è sempre pronto a morire e rasenta continuamente il sottile crinale che separa l'essere dal nulla.

Con i suoi slanci rapinosi e i suoi terrori, le sue estasi e le sue violente liberazioni di forze, la guerra seguita ad essere, nel regno del fake e dei simulacri digitali, una delle rare forme di sconcertante genuinità che sia dato di esperire. Quando la paura morde all'addome e i corpi vulnerati prendono a spicciare sangue, si dissolvono all'istante i filtri dell'effetto reality e si ritorna alla cruda verità della carne. È difficile negare autenticità a un corpo che sanguina. Dinanzi al pericolo assoluto tutto si essenzializza; nulla di ciò che governa la realtà profana serba il minimo valore e per converso tornano a contare i beni assoluti: la nuda vita e l'onore. Sotto la minaccia della morte, ogni momento si carica di attesa, perché potrebbe essere l'ultimo e il decisivo. Lo scatenamento della violenza e del conflitto acuisce la percezione del vivere, perché accosta l'uomo all'irreparabile, alle scelte postreme, agli eventi definitivi e senza ritorno. Il combattimento è, per eccellenza, il luogo dell'inappellabile. Dentro l'orizzonte bellico, la sensibilità e l'agire si de-automatizzano: si rompe la routine del sempre eguale. All'esercizio piattamente iterativo dei gesti quotidiani si sostituisce l'unicità dell'atto perentorio e irripetibile, che spacca e ferisce, separando irrevocabilmente il prima dal poi. Così, la guerra svela e verifica: è uno stato d'eccezione che permette di cogliere l'anima del mondo. Battendosi per vincere o morire, si torna alle origini, ai segreti della spontaneità primeva: tutto riacquista i sapori forti della "prima volta”, gli aromi stordenti dei primordi. In molte culture guerriere, si ritiene che la realtà fisica e materiale dello scontro possa condurre a stati spirituali così tesi ed e sottili, da schiudere varchi inaspettati verso piani di esistenza più elevati. La danza travolgente $\mathrm{e}$ feroce della battaglia ci integra in un modo di esistere amplificato, più ritmato del normale, più scandito dell'usuale, e questa condizione "drogata" ci proietta verso una vita "in maggiore”, ad altissimo voltaggio e alle massime frequenze. Il conflitto è il luogo del massimalismo esistenziale: tutti i sensi vi si trovano dilatati e tesi al diapason. La guerra, avvicinando l'uomo ai territori extra-ordinari del sacro, si offre dunque come una modalità di esistenza di ordine superiore, un'esuberanza di vita, un trabocco energizzante di forze prorompenti, uno stordente contatto con dimensioni supreme, eccedenti la normalità del quotidiano e della gente comune. A riscontro di queste considerazioni, alle- 
Vivere per morire o morire per vivere: l'archetipo della morte precoce nelle tradizioni eroiche

ghiamo un frustolo di cruda e luminosa verità testimoniale dedotto dalla prosa sovreccitata ma meravigliosamente lucida di Ernst Jünger:

Già, questo incantesimo delle armi lampeggianti, del sangue schiumante e del gioco audace per la vita e per la morte sembrava superare di gran lunga ciò che l'esistenza aveva altrimenti da offrire. ${ }^{5}$

La guerra è l'esistenza scritta in caratteri maiuscoli, ${ }^{6}$ affettivamente survoltata ed esperita a un livello emozionale superlativo, e per questo motivo è la specola ideale per osservare i misteri angosciosi della morte, gl'incanti del mondo e il desiderio degli individui di sopravvivere o di perpetuarsi nel ricordo.

In battaglia il tempo ha una qualità speciale, diversa dall'ordinario: da un lato, tutto sembra procedere più in fretta, per strappi e brusche accelerazioni; dall'altro lato, il trascorrere veloce e ritmato delle azioni può inciampare in momenti di ristagno e risacca. Il contatto con la morte e il terrore del dissolvimento propiziano sobbalzi emozionali ed effetti di alterazione percettiva che producono violente distorsioni del tempo. È la «giostra dei sensi $\gg^{7}$ che tanti Combat movies hollywoodiani si incaricano di restituire per il tramite di riprese in soggettiva, ralenti enfatico, trattamenti accelerati, montaggio adrenalinico, effetti di sgranato o di flou alternati a immagini di allucinatoria esattezza iperrealistica: tutti mezzi stilistici che certo avranno di mira il gusto fracassone di un pubblico dal palato grosso, ma che indubitabilmente riescono nell'intento di mimare l'esperienza immersiva e frastornante del combattimento, con i suoi esiti di disorientamento e di vertigine. Come avviene per l'eccitazione dell'eros, la natura orgasmica della guerra promuove forme di affettività parossistica che sono proprie ai picchi dell'esistenza.

${ }^{5}$ JÜNGER, Fuoco e sangue: 10-11.

${ }^{6}$ Cfr. VAN CREVELD 1998: 286.

${ }^{7}$ Deduco il sintagma dal titolo di un'opera composita di Ardengo Soffici (cfr. SoffICI, La giostra dei sensi), costruita a mo' di collage come montaggio di bozzetti, aneddoti, pensieri, aforismi, strofette giocose, pagine di journal intime e piccole trovate. L'intestazione di questo album appare oltremodo calzante, perché nei frammenti che lo compongono vibra una sensualità vertiginosa e intensificata, nutrita di note aggressive e di languido erotismo. Coi suoi movimenti centrifughi, le sue intemperanze e il suo gusto del bizzarro, il testo di Soffici convoca una realtà ora misteriosa e velata, ora oscenamente esibita, un demi-monde dove si muovono «guaglione splendenti e scugnizzi» (ivi: 7), soldati e donne facili. 
L'Erlebnis della battaglia obbedisce a una temporalità slogata e sussultante: ${ }^{8}$ un asincronismo intermittente che si comprime nell'ipercinesi, ma che può dilatarsi in vaste sospensioni durante le quali il soggetto attonito si confronta con una rivelazione carica di destino o, tutto all'opposto, contempla la desolazione di un mondo incomprensibile e svuotato di senso.

Si è detto che la battaglia è prova suprema ${ }^{9}$ e, insieme, una bisca tremenda in cui si punta il bene più grande, il posto in cui ognuno gioca a "tutto o niente", scommettendo la propria persona e impegnando per intero sé stesso. In tale prospettiva, il combattimento non è mai una lunga partita a scacchi con la morte, ma un lancio di dadi perentorio, di quelli che stabiliscono le sorti una volta per tutte e senza appello. A minacciare l'uomo in armi non è l'inerzia logorante, l'avvizzimento dell'età che anno dopo anno rammollisce i corpi, lasciandoli flosci e affraliti, ma il colpo saettante vibrato nell'istante risolutivo, nel momento supremo che innalza agli onori o precipita nell'infamia. Per molti aspetti la cosa più interessante, nella vita, è la morte, ma a questo assunto bisognerebbe subito allegare una precisazione: nelle culture di guerra e nelle civiltà di vergogna, ciò che veramente vale e si carica di senso non è il decesso in sé, ma il modo in cui si muore, imprimendo alla propria vita uno stigma definitivo. Una fine nobile - con le armi in pugno e acie aperta -, che tronca una vita traboccante di energie, recisa proprio al colmo del suo impetuoso movimento espansivo: è questa, nell'oltranzismo del combattentismo epico, l'apoteosi del guerriero. Entro tale concezione si affida al trapasso, in quanto punto di culminazione di una parabola destinale, il compito di perfezionare la vita, facendo degli individui ciò che realmente sono. È una sorta di rovesciamento paradossale, per cui la morte non è più l'agente del nulla, che dissolve la persona e la sprofonda nel non essere, ma l'occasione superlativa, l'appuntamento col destino che muta l'uomo in sé stesso e dà significato alla sua esistenza proprio nel momento in cui la

\footnotetext{
${ }^{8}$ Ecco un altro paradosso di quello stato d'eccezione che è la guerra combattuta: da un canto la temporalità degli scontri armati è fatta di distorsioni percettive, di precipitose accelerazioni e larghi ristagni; dall'altro canto, gli uomini immersi nel furore della battaglia possono provare, in circostanze particolari, la sensazione di raggiungere il Grande Tempo, installato nell'assolutezza di un presente immobile o in un riflusso di sospensione atemporale. A volte, proprio nella violenza della mischia, si fa esperienza del tempo dei tempi, di un modo d'essere cronologicamente illimitato e non condizionato, estraneo al divenire storico e sottratto alla successione consequenziale degli accadimenti.
}

${ }^{9}$ Cfr. Benoist 1999: 72-74. 
Vivere per morire o morire per vivere: l'archetipo della morte precoce nelle tradizioni eroiche

estingue: «Tel qu'en Lui-même enfin l'éternité le change». ${ }^{10}$ Più in particolare, la fine dell'eroe si carica di valenze superiori soltanto quando si cristallizza in forme monumentali, facendo del transeunte e dell'accidentale un essere-una-volta-per-tutte. ${ }^{11}$ Per risultare efficace come dispositivo eternante e quale produttore di prestigio, la morte deve rappresentare il campione nella sua interezza, ovvero rinchiudere tutta la sua vita e le sue qualità in un solo istante risolutivo di fiammeggiante tensione. Nell'andare consapevolmente verso la morte, il combattente compie un risoluto atto di soggettivazione, rivendicando piena signoria sulla propria vita e giungendo ad essere realmente $d i$ sé stesso. Ma ciò che sembra davvero essenziale nelle società di vergogna è la volontà dell'eroe di costruirsi una fine gloriosa, la ferma intenzione di cadere con onore, così da assicurarsi fama e rinomanza. I grandi campioni militari celebrati dall'epica universale temono assai più il disonore della morte: a Roncisvalle, Orlando non pensa a come salvarsi né teme di soccombere alle soverchianti forze saracene, ma è ossessionato dal timore di perdere la faccia e trema all'idea che aedi e giullari possano cantare di lui e dei suoi uomini una canzone di biasimo e di infamia: ${ }^{12}$

Ben devuns ci estre pur nostre rei:

Pur sun seignor deit hom susfrir destreiz

E endurer e granz chalz e granz freiz,

Sin deit hom perdre e del quir e del peil.

Or guart chascuns que granz colps [i] empleit,

$\mathrm{Mal}[\mathrm{e}]$ cançun de nus chantét ne seit!

${ }^{10}$ Stéphane Mallarmé, Le tombeau d'Edgar Poe, in MALLARMÉ, Poesie: 148 . Il decesso sul campo di battaglia è il più violento e accelerato dei processi trasformativi: con la sua torsione subitanea, esso fa emergere dalla latenza le virtù del guerriero e le eterna in un gesto di assoluta evidenza. La morte rossa fa sì che l'eroe possa diventare realmente sé stesso.

${ }^{11}$ La morte gloriosa in combattimento non soltanto sospinge all'estremo l'intensità della vita, conducendo a un veemente potenziamento delle percezioni, ma si configura come il momento sommamente rivelativo dell'esistenza: la sostanza dell'eroe si disvela nella sua autenticità solo nelle fasi drammatiche del trapasso, quando i valori e gli ideali vengono al pettine della paura e del sangue.

${ }^{12}$ Canzone di Orlando [Segre], vv. 1009-1014. Nelle società militari dell'onore, la memoria tribale della prodezza guerriera è affidata al canto di aedi, bardi e rapsodi, che compongono inni di encomio e di lode per celebrare le magnifiche imprese degli eroi, stigmatizzando e biasimando per converso le condotte pusillanimi e inadeguate. 
'Dobbiam restare qui per il nostro re: / giusto è soffrire, se al proprio signor serve, / e sopportare gran caldo e grande freddo: / perfino pelle, perfin capelli perderci! / Or d'assestare gran colpi ognuno cerchi, / perché non dicano di noi canzon di scherno!'

\section{A CERCAR LA BELLA MORTE: LA BATTAGLIA COME DISPOSITIVO GLORIFICANTE E MACCHINA DELL'IMMORTALITÀ}

Non c'è bella morte senza vita breve (Jean-Pierre Vernant, La morte eroica nell'antica Grecia)

Il luogo e il tempo della battaglia sono quelli in cui più si avverte la precarietà della vita umana, con i suoi tratti di limitatezza e fragilità, ma sono anche le coordinate che definiscono il paradigma della gloria e della trasfigurazione eroica. Il più micidiale dispositivo di annientamento è anche la più formidabile macchina dell'immortalità. Spetta agli antropologi del mondo greco - e particolarmente a Jean-Pierre Vernant ${ }^{13}$ - il merito di aver mostrato l'efficacia della "bella morte" come strumento rituale per sfuggire all'usura del tempo tramite la costruzione di un kléos imperituro. La fine eroica è la risposta del guerriero alla corruttibilità della carne. Estinguersi in modo memorabile - morire la bella morte - è il sistema con cui i campioni omerici tentano di contrapporsi drammaticamente alla caducità umana, riscattando la labilità della vita col potere eternizzante della fama militare. La morte anzitempo ricevuta in battaglia non soltanto ha il potere di perpetuare l'eroe nella memoria e nell'epos, ma sottrae l'illustre defunto al degrado del corpo, che a poco a poco nei vivi perde smalto e deperisce, fino a sfiorire nella decrepitezza della penosa vecchiaia. Al declino biologico e alla lenta decadenza fisica l'eroe contrappone la morte veloce e violenta - sul campo, seguita dal perdurare del suo ricordo nel sapere condiviso dell'enciclopedia tribale. L'uomo, essere transeunte e soggetto alla tirannia del divenire, non può farsi immortale come gli dèi, ma con le sue gesta è in grado di ottenere una sorta di trionfo glorificante che gli garantisce un surrogato di eternità. Al rodio del tempo, che lento ma inesorabile mastica e allenta la carne, egli sostituisce la morte prematura nel

${ }^{13}$ Cfr. VernANT 2019: passim, ma soprattutto alle pp. 8, 11-13, 20-21, 24-25. 
Vivere per morire o morire per vivere: l'archetipo della morte precoce nelle tradizioni eroiche

furore della lotta e nello splendore della forza. Il furore della guerra distrugge istantaneamente, in un corrusco balenio di ferro e di sangue, le belle membra giovanili che l'età si sarebbe incaricata di umiliare con un lento scivolamento nella decrepitezza. L'azione devastatrice delle lame da un lato fa scempio dei corpi, ma dall'altro lato li sottrae alla corruttibilità, affrancandoli dagli effetti dell'invecchiamento. Lo squarcio della ferita è deturpante, ma regala al caduto una forma di attualizzazione assoluta, fuori dal tempo, fissando un destino individuale nella gloria di un gesto finale degno di memoria. In tal modo le armi, nel barbaglio trafiggente dell'affondo, svolgono un ufficio feroce e insieme pietoso. Cadere nelle prime file mentre si è ancora nel fiore dell'età significa eludere l'autunno della vita, restando per sempre nella primavera gioiosa di un corpo fresco e splendente di forza. In tal modo, i ragazzi deceduti in battaglia rimangono giovani per sempre, continuando ad essere, anche da trapassati, ciò che erano da vivi. ${ }^{14} \mathrm{~L}$ 'istituto della morte eroica, elaborato dalla cultura militare aristocratica dell'antica Grecia, innerva in profondità la condotta degli eroi omerici e si fissa con la pervasività di un riferimento ideale nella tradizione occidentale, tanto da divenirne un luogo comune ricorsivo, talora arricchito di implicazioni sacrificali, talaltra tecnicizzato e piegato a esigenze di propaganda. Il radicamento e la persistenza di questo costrutto ideologico sono documentati anche di riflesso, dall'impegno con cui le poetiche antimilitariste della modernità si sono incaricate di decostruire e demistificare il tópos della bella morte, facendo reagire la tanatofilia delle pose belliciste con l'atroce macello dei corpi precipitati nella guerra dei mezzi e dei materiali. Ciononostante, non sono mancate, nel cuore di tenebra del secolo di ferro, clamorose riattivazioni del motivo. Si veda, ad esempio, in che modo lo schema

\footnotetext{
${ }^{14}$ Il decadimento e l'invecchiamento possono essere superati e sublimati facendo della morte - la morte rossa e violenta, guerresca e cruenta - la posta suprema, il valore assoluto e il punto di culminazione di una vita esemplare. Il trapasso eroico si presenta allora come un meccanismo glorificante che viene giocato contro la precarietà e la finitudine: è un modo di riscattare e di trascendere, per il tramite del valore guerriero, il transitorio e l'effimero, ossia la natura statutariamente passeggera della condizione umana. Morire da prodi in combattimento non vuol dire soltanto fissare la propria immagine in un profilo illustre e degno di memoria: significa anche preferire una fine subitanea nel fiore degli anni alla lenta corruzione che lavora all'interno dei corpi, logorandoli e facendoli avvizzire, privandoli a poco a poco del fulgore e della bellezza giovanili. L'età usura le fibre, deteriora e degrada. Morire bene - cioè cadere gloriosamente in battaglia - è un modo per sconfiggere la mortalità che svuota, rammollisce e disfa gradualmente, giorno dopo giorno, la carne degli uomini. La "bella morte" completa e sigilla la biografia dei grandi campioni militari, fissando il combattente nel fulgore di una giovinezza definitiva e inalterabile. Cfr. al riguardo VERNANT 2019: 35-37, 41, 51, 88.
} 
ritorni sotto la penna di Umberto Saba, scandito con un'intonazione misticheggiante che valorizza la morte precoce come compimento di una comunione spirituale ed eterna con il gruppo ristretto della comunità virile:

poi che la vita è un male, e son moleste, dopo la prima giovinezza, l'ore:

ma chi soldato fra i soldati muore, resta giovane sempre sulla terra. ${ }^{15}$

La tensione dei campioni militari verso la fama, il loro bisogno di conquistare le luci della ribalta, la loro smania di sottrarsi all'oblio attraverso imprese d'eccezione e perciò memorabili: tutte queste invarianti dell' ethos eroico sono riportabili al paradigma della lotta con il caos e con l'amorfo. In un certo senso, non si dà azione dei combattenti d'élite che non sia interpretabile nei termini di una strenua militanza contro il preformale e contro le forze regressive, che fanno scivolare il mondo nell'indistinto e nelle latebre del non-essere. Difensore e custode dell'integrità tribale, l'eroe è dalla parte dell'ordine, dell'armonia regolata e della gerarchia: ogni suo gesto mira a contrastare gli impulsi che spingono il mondo verso il ritorno all'informe e all'indifferenziato. I super-guerrieri delle società arcaiche e dell'epica universale:

1) aspirano a singolarizzare le loro prestazioni, tendono cioè a individualizzare l'exploit, personalizzandolo e definendolo, staccandolo dall'indifferenziazione dei grandi numeri e dal brulichio confusivo delle masse; ${ }^{16}$

\footnotetext{
${ }^{15}$ Umberto Saba, Congedo (estraggo la citazione da CORTELLESSA 2018: 125).

${ }^{16} \mathrm{~L}$ 'isolamento ingrandisce ed esalta il valore eroico: per questo i fuoriclasse e i personaggi di primo piano si sfilano dalle schiere e corrono incontro al nemico per ingaggiare sfide individuali. Una delle più iconiche messe in scena di uno scontro tra capi, preliminare al combattimento collettivo tra due armate, si legge ne $\mathrm{La}$ conquête de Constantinople di Robert de Clari (il fatto d'arme risale al 1187). Pochi istanti prima che le loro formazioni impattino violentemente, dando inizio alla mischia generale, il marchese Corrado di Monferrato e Alessio Brana escono dai ranghi e si affrontano in duello: «Si comme il venoient, si ne fait mais el li Vernas, si se fiert il des esperons, si se met il devant toute se gent bien le getee d'un cailleu, pour soi haster et pour ferir soi en le batalle le marchis. Quant li marchis le vit venir, si point il encontre lui, si le fiert il au premerain coup en l'uel, si l'abat il mort de chu caup; si fiert a destre et a senestre, et il et se gent, si en ochient mout» ('Mentre correvano gli uni contro gli altri, Brana non perse certo tempo, ma spronò il cavallo tanto da spingersi davanti
} 
Vivere per morire o morire per vivere: l'archetipo della morte precoce nelle tradizioni eroiche

2) cercano spazi e occasioni di visibilità sul campo di battaglia, smarcandosi dal grigiore delle schiere e assicurando alle loro imprese una collocazione solitaria, in piena luce; ${ }^{17}$

3) sottraggono la prestazione militare all'insignificanza e all'ombra del nulla, per farne la base di una memoria identitaria, di un senso di appartenenza etnica e di un epos tribale;

ai suoi di un buon tiro di pietra: voleva aumentare l'andatura e piombare sulle schiere del marchese. Questi, vedendolo venire, si diresse contro di lui e lo centrò al primo assalto in un occhio, abbattendolo morto al suolo; egli e la sua gente presero poi a colpire a destra e a manca, facendo grande strage'; ROBERT DE CLARI, La conquête de Constantinople [Lauer], cap. XXXIII, 11. 60-67: 33-34). La singolar tenzone tra Corrado di Monferrato e Brana è riferita anche in un passo parallelo di ConIATA, Grandezza e catastrofe di Bisanzio [Pontani - van Dieten], XII 10, 21: 287. La tenzone singolare disputata a mo' di amuse-bouche, nell'imminenza dello scontro generale, mette in scena per il solito campioni affermati, condottieri in vena di strafare oppure giovani avidi di gloria che escono dai ranghi e vanno a battersi nella terra di nessuno posta tra due armate contrapposte e schierate in campo aperto. Questi duelli “incipitari" - talvolta interpretati a mo' di vaticinio per preconizzare l'esito del susseguente scontro di massa - sono riservati ai combattenti di élite che vogliono mettersi in mostra cercando l'azione isolata e il coup d'éclat. Insofferenti alla disciplina collettiva, i primi feditori combattono malvolentieri nella massa e cercano in ogni modo di mettersi in mostra.

${ }^{17}$ Smaniosi di segnalarsi e di fare spicco, i guerrieri d'alto grado compiono ogni sforzo per occupare il centro della scena: si collocano nel punto di massima esposizione, sotto le luci della ribalta, e tentano di intercettare tutti gli sguardi. I grandi campioni hanno un senso istintivo della teatralità: non sopportano i bassifondi della battaglia, soffrono la mediocrità dei contesti ordinari, prediligono l'assolo, cercano l'azione isolata e solitaria che dà lustro al valore individuale. L'eroe è colui che sa emergere dall'ombra anonima della mischia per sollevarsi alla luce della gloria: la sua prodezza trova senso solo nell'ostensione e nell'esibizione spettacolare. Nelle culture di vergogna, non c'è distinzione tra essenza e apparenza: l'esteriorità è sostanza e la fama si consegue solo attraverso il riconoscimento e l'apprezzamento generali. Entro una cultura siffatta, l'unico eroismo possibile è quello esteriore e manifesto, legittimato da tutti e socialmente validato. Per le società dell'onore e del faccia a faccia, il valore e la sostanza di ogni uomo non rimandano alla soggettività o all'auto-percezione, ma si definiscono attraverso lo sguardo degli altri: l'esistenza di ciascuno è posta sotto gli occhi giudicanti della collettività. Mediante le sue prestazioni marziali - eseguite apertamente e coram populo -, il guerriero modella la propria immagine. La costruzione del suo profilo sociale si compie tramite la realizzazione di imprese e atti di coraggio che devono ricevere l'approvazione collettiva, in accordo con un sistema di riferimenti codificati e uno standard di condotta largamente condiviso. Le virtù e il pregio di un campione divengono reali solo quando sono dimostrati alla luce del giorno: in maniera inequivocabile e di fronte a tutti. La necessità di una ratifica pubblica conferisce alla visibilità dell'exploit militare una funzione essenziale. Oltre che maiuscolo e notevole, il gesto guerriero dev'essere evidente, lampante e perentoriamente esibito: uno sfolgorante assolo eseguito in pieno sole. Per le civiltà di vergogna, l'“essere per sé" e la coscienza individuale della propria bravura non possono bastare, perché sono l'esteriorità e la fama a dare forma alla personalità sociale, determinando la misura del valore. Sul paradigma della visibilità nel discorso bellico e nelle narrative di guerra del mondo occidentale, si leggano le sintesi di Antonio Scurati: SCURATI 2003; SCURATI 2006: 17-21; SCURATI 2016: 32-41. 
4) muoiono giovani, nel fiore della virilità, e con la loro morte prematura tolgono potere all'opera corruttrice e disgregatrice del tempo, che nel corso degli anni infrollisce la carne e sconcia la bellezza degli uomini.

Sul piano del pensiero arcaico, non è difficile scorgere l'omologia di struttura sottesa a queste linee di condotta. Ciò che le unifica è la contrapposizione combattiva all'indeterminatezza e all'indistinto. I "nemici” dell'eroe sono il rimenio confuso e il disordine magmatico della mischia, che inghiotte e cancella nella spessa calca il valore personale; l'anonimato del collettivo, ${ }^{18}$ che minaccia la luminosità stagliata e individuale del gesto guerriero; l'oblio e l'oscurità, che attentano alla fama delle imprese memorabili; il lavorio inesorabile del tempo, che consuma i corpi avviandoli lentamente alla regressione larvale o all'abisso del nulla.

\section{COME LE FOGLIE}

Quel che contestavo [...] era il pessimismo [...] per cui il carattere effimero del bello comporterebbe la sua radicale svalorizzazione. $\mathrm{Al}$ contrario, un aumento del suo valore! Il valore di caducità è un valore di rarità nel tempo. Il limite imposto alla possibilità del godimento non fa che impreziosirlo.

(Sigmund Freud, Caducità)

Dalla poetica della caducità di Mimnermo all'Imitazione leopardiana della Feuille di A.V. Arnault ${ }^{19}$ il paragone della «povera foglia frale» ha attraversato l'intero corso della lirica occidentale, affidando all'immagine-emblema del ciclo vegetale il discorso sull'impermanenza e sullo scivolio inesorabile delle generazioni lungo la nera schiena del tempo. Più in particolare, all'interno di questa costellazione, si delinea un filone specifico di simi-

\footnotetext{
${ }^{18}$ L'eroismo consiste anzitutto nell'affermazione di un'individualità. Mediante l'unicità delle sue imprese, il grande campione si fa un nome: esce dal grigiore dell'anonimato senza onore, si smarca dall'omologazione della folla. L'azione isolata permette al fuoriclasse di emergere dall'ombra, lo stacca dall'insignificanza del gruppo. I primi ballerini della guerra, i divi delle schiere, le vedettes dei teatri di Ares pretendono le luci della ribalta e sono insofferenti alla mediocrità delle seconde file. Si legga, in proposito, CAU 2013: 61.

${ }^{19}$ Cfr. LeOpARDI, Canti: 325-327.
} 
Vivere per morire o morire per vivere: l'archetipo della morte precoce nelle tradizioni eroiche

litudini che esprimono la vulnerabilità della condizione umana esposta alla violenza immane della guerra. Dal dialogo di Glauco e Diomede nel libro VI dell'Iliade all'angoscia esistenziale dei Soldati di Ungaretti, ${ }^{20}$ immersi nelle tempeste d'acciaio del Primo Conflitto Mondiale, diviene quasi inevitabile comparare la gracilità creaturale degli uomini in guerra con la transitoria cedevolezza delle foglie trascinate dal vento. E forse, fra le tante riprese epiche e liriche, una delle più alte esecuzioni del motivo si deve a Giani Stuparich, che fa risuonare questa immagine di diffusione universale nel referto asciutto e onesto del suo diario di guerra: ${ }^{21}$

Una grande pietà mi prende di questa povera carne, di me stesso, così piccolo e debole. Tanto decisi, tanto pronti a morire e ad uccidere: e, in fondo, come le foglie sbattute dall'uragano.

Eppure, eppure. A fianco di questo immaginario dolente, in cui le sorti precarie dei guerrieri sono assimilate alla labilità delle foglie, si profila potente un'altra serie topica di ispirazione vegetale, che si connota invece in senso euforico, costruendo la bellezza lampeggiante della gloria militare proprio sui prestigi dell'effimero. Penso, ovviamente, ai tanti esordi stagionali che risuonano dal fondo del Medioevo neolatino, tra la lirica dei trovatori e le canzoni di gesta in lingua d'oül, dove la primavera delle passioni si confonde con la primavera di prodezza dei fatti d'arme, secondo un intreccio davvero archetipico di Ares e di Eros. ${ }^{22}$ In questi testi, il cronotopo della guerra di primavera s'incarica di

${ }^{20}$ Soldati (1918), ultimo testo della sezione Girovago de L'Allegria, propone quella che mi pare la più epigrafica e proverbiale esecuzione di questa topica comparazione: un vero pezzo da antologia (cfr. UNGARETTI, Vita d'un uomo [Piccioni]: 87). L'affinità uomo-foglia come espressione di finitudine terrena e fragilità creaturale ritorna nel pezzo d'apertura di Sentimento del tempo: «Foglie, sorelle foglie, / Vi ascolto nel lamento» (O notte, vv. 4-5; cfr. ivi: 103).

${ }^{21}$ STUPARICH, Guerra del '15 [Sandrini]: 175.

${ }^{22}$ Si pensi, per restringersi a un solo esempio trascelto dall'ambito occitanico, al sirventese Be m platz lo gais temps de pascor (BdT 80, 8a), capolavoro dell'esiguo filone del "combattentismo trobadorico", su cui si veda soprattutto MANCINI 1993: 133-161, che sottolinea opportunamente l'intreccio inestricabile di plazer e enueg, di gioiose impennate dei sensi e foschi emblemi di morte. Tutto il componimento è costruito su una testura di termini semanticamente carichi, che accosta e pone in tensione la selvaggia felicità marziale della cavalleria e il senso di angoscia indotto dalla contemplazione delle armi e dei corpi fatti a pezzi. Il rovinio dell'equipaggiamento spaccato, lo scempio della carne offesa, i cavalli scossi che corrono impazziti di paura per il campo di battaglia compongono un diorama di morte che ispira sentimenti disforici e luttuosi, ma è 
ricordarci che, per le società premoderne, le attrattive della giovinezza amorosa e guerriera sono indissociabili dal sentimento della loro estrema fugacità, e che insomma la bellezza è tanto più inarrivabile quanto più è fuggevole. Siamo nell'alba romanza della tradizione europea, nel cuore tenero e ferrigno dell'ideologia cavalleresca e della società feudale, ma non ci troviamo poi cos̀̀ lontani dalla poetica dei fiori di ciliegio del Giappone dei samurai. ${ }^{23}$

anche un magnifico spettacolo di forza nel quale vibra un esaltante e gioioso furore, una selvaggia felicità marziale capace di intonarsi agli stessi timbri squillanti su cui si accordano le espressioni del trasporto amoroso. L'arrivo della buona stagione risveglia i sensi e in pari tempo ridà fiato e slancio alle attività militari: col bel tempo si riaccendono le passioni e ritorna la galloria delle allegre carole, ma riprendono anche le cavalcate di primavera, le festose gualdane e i raids di saccheggio. Per una visione più generale sulla primavera d'armi, tra euforia desiderante e "arditismo" cavalleresco, cfr. BARBIERI 2016. La poesia delle armi, come quella d'amore, è sempre una "canzone del maggio".

${ }^{23}$ Sulla predilezione tipicamente nipponica per ciò che è passeggero e transeunte si veda l'eccellente sintesi di Marcello Ghilardi in questo stesso fascicolo di «AOQU Achilles Orlando Quixote Ulysses» (La morte dell'eroe nella tradizione giapponese). Il pathos creaturale per ogni cosa che cade è un motivo ricorrente del pensiero giapponese, così come la commozione struggente per tutto quanto vive di vita effimera, costeggiando di continuo l'ombra della morte e il crinale del nulla. 


\section{BIBLIOGRAFIA}

BIBLIOGRAFIA PRIMARIA

Canzone di Orlando [Segre] = La Canzone di Orlando, introduzione e testo critico di Cesare Segre, traduzione di Renzo Lo Cascio, premessa al testo, note e indici di Mario Bensi, Milano, Rizzoli, 1996.

CONIATA, Grandezza e catastrofe di Bisanzio [Pontani - van Dieten] = Niceta Coniata, Grandezza e catastrofe di Bisanzio, Vol. II, a cura di Jan-Louis van Dieten - Anna Pontani, Milano, Fondazione Lorenzo Valla - Mondadori, 2001.

CORTELLESSA 2018 = Le notti chiare erano tutte un'alba. Antologia dei poeti italiani nella Prima guerra mondiale, nuova edizione accresciuta, a cura di Andrea Cortellessa, Milano, Bompiani, 2018 [I ed. Milano, Bruno Mondadori, 1998, prefazione di Mario Isnenghi].

JÜNGER, Fuoco e sangue = Ernst Jünger, Fuoco e sangue. Breve episodio di una grande battaglia, Milano, Guanda, 2016 [ed. or. Feuer und Blut. Ein kleiner Ausschnitt aus einer grossen Schlacht, 1925].

LEOPARDI, Canti [Bandini] = Giacomo Leopardi, Canti, introduzione, commenti e note di Fernando Bandini, Milano, Garzanti, 1988.

MALlarmé, Poesie = Stéphane Mallarmé, Poésies/Poesie, Milano, Feltrinelli, 1980.

ROBERT DE ClARI, La conquête de Constantinople [Lauer] = Robert de Clari, La conquête de Constantinople, éditée par Philippe Lauer, Paris, Champion, 1956.

SOFFICI, La giostra dei sensi = Ardengo Soffici, La Giostra dei Sensi, Firenze, Vallecchi, $1920^{2}$ [I ed. Firenze, Edizioni della Voce, 1918].

STUPARICH, Guerra del '15 [Sandrini] = Giani Stuparich, Guerra del '15, a cura di Giuseppe Sandrini, Macerata, Quodlibet, 2015.

UNGARETTI, Vita d'un uomo [Piccioni] = Giuseppe Ungaretti, Vita d'un uomo. Tutte le poesie, a cura di Leone Piccioni, Milano, Mondadori, 1992. 


\section{BIBLIOGRAFIA SECONDARIA}

BARBIERI 2016 = Alvaro Barbieri, "Era de maggio": tópos primaverile e poetica della guerra nella letteratura cavalleresca del Medioevo di Francia, in Amb. Dialoghi e scritti per Anna Maria Babbi, a cura di Giovanni Borriero - Roberta Capelli Chiara Concina - Massimo Salgaro - Tobia Zanon, Verona, Fiorini, 2016, 65-74.

BENOIST 1999 = Alain de Benoist, Ripensare la guerra. Dallo scontro cavalleresco allo sterminio di massa, Milano, Terziaria, 1999.

CAILLOIS 2001 = Roger Caillois, L'uomo e il sacro, a cura di Ugo M. Olivieri, Torino, Bollati Boringhieri, 2001 [ed. or. L'Homme et le sacré, Paris, Gallimard, 1950].

CAU 2013 = Jean Cau, Il Cavaliere, la Morte e il Diavolo, prefazioni di Pietrangelo Buttafuoco - Sigfrido Bartolini, Roma, Settimo Sigillo, 2013 [ed. or. Le Chevalier, la Mort et le Diable, Paris, La Table Ronde, 1977].

ELIADE 1968 = Mircea Eliade, Il mito dell'eterno ritorno (Archetipi e ripetizione), Milano, Borla, 1968 [ed. or. Le mythe de l'éternel retour - Archétypes et répétition, Paris, Gallimard, 1949].

MANCINI 1993 = Mario Mancini, Metafora feudale. Per una storia dei trovatori, Bologna, il Mulino, 1993.

SCURATI 2003 = Antonio Scurati, Guerra. Narrazioni e culture nella tradizione occidentale, Roma, Donzelli, 2003.

SCURATI 2006 = Antonio Scurati, La guerra come rappresentazione rassicurante, in Conflitto e narrazione. Il racconto della guerra nella società della comunicazione di massa, a cura di Vittorio Mathieu, Bologna, il Mulino, 2006, 11-53.

SCURATI 2016 = Antonio Scurati, Dal tragico all'osceno. Raccontare la morte nel XXI secolo, Milano, Bompiani, 2016.

VAN CREVELD 1998 = Martin Van Creveld, La transformation de la guerre, Monaco, Éditions du Rocher, 1998.

VERNANT 2000 = Jean-Pierre Vernant, L'individuo, la morte, l'amore, edizione italiana a cura di Giulio Guidorizzi, Milano, Raffaello Cortina Editore, 2000 [ed. or. L'individu, la mort, l'amour, Paris, Gallimard, 1989]. 
Vivere per morire o morire per vivere: l'archetipo della morte precoce nelle tradizioni eroiche

VERNANT 2019 = Jean-Pierre Vernant, La morte eroica nell'antica Grecia, a cura di Simone Regazzoni, Genova, Il nuovo melangolo, 2019 [ed. or. La mort hérö̈que chez les Grecs, Nantes, Éditions Pleins Feux, 2001]. 